\title{
Messages on pregnancy and family planning that providers give women living with HIV in the context of a Positive Health, Dignity, and Prevention intervention in Mozambique
}

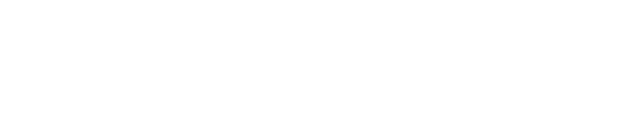

Starr Hilliard

Sarah A Gutin

Carol Dawson Rose

Department of Community Health Systems, School of Nursing, University of California at San Francisco, San Francisco, CA, USA
Correspondence: Starr Hilliard Department of Community Health Systems, School of Nursing, University of California at San Francisco,

2 Koret Way, Room N505, Box 0608,

San Francisco, CA 94143, USA

$\mathrm{Tel}+\mathrm{I} 9256998778$

Fax + I 4154766042

Email starrhilliard@gmail.com
Background: Family planning is an important HIV prevention tool for women living with HIV (WLHIV). In Mozambique, the prevalence of HIV among women of reproductive age is $13.1 \%$ and the average fertility rate is high. However, family planning and reproductive health for WLHIV are under-addressed in Mozambique. This study explores provider descriptions of reproductive health messages in order to identify possible barriers and facilitators to successfully addressing family planning and pregnancy concerns of WLHIV.

Methods: In 2006, a Positive Health, Dignity, and Prevention program was introduced in Mozambique focused on training health care providers to work with patients to reduce their transmission risks. Providers received training on multiple components, including family planning and prevention of mother-to-child transmission (PMTCT). In-depth interviews were conducted with 31 providers who participated in the training in five rural clinics in three provinces. Data were analyzed using qualitative content analysis.

Results: Analysis showed that providers' clinical messages on family planning, pregnancy, and PMTCT for WLHIV could be arranged along a continuum. Provider statements ranged from saying that WLHIV should not become pregnant and condoms are the only valid form of family planning for WLHIV, to suggesting that WLHIV can have safe pregnancies.

Conclusion: These data indicate that many providers continue to believe that WLHIV should not have children and this represents a challenge for integrating family planning into the care of WLHIV. Also, not offering WLHIV a full selection of family planning methods severely limits their ability to protect themselves from unintended pregnancies and to fully exercise their reproductive rights. Responding to the reproductive health needs of WLHIV is a critical component in HIV prevention and could increase the success of PMTCT programs.

Keywords: prevention of mother-to-child transmission, provider messages, stigma, family planning, women, human immunodeficiency virus

\section{Introduction}

In Mozambique, there are 1.4 million people living with HIV (PLHIV), and $11.5 \%$ of adults who are in their reproductive years (aged 15-49 years) are HIV-positive. ${ }^{1}$ The prevalence rate among women is even higher at $13.1 \% .{ }^{1}$ In $2009,77 \%$ of pregnant women received HIV testing and counseling. ${ }^{2}$ Of the 97,000 pregnant women living with HIV (WLHIV), 70\% received antiretroviral therapy (ART) to prevent vertical transmission, or as it is known globally, prevention of mother-to-child transmission (PMTCT). ${ }^{2}$ The percentage of PLHIV receiving ART for their own health remains low. 
As of $2009,12.2 \%$ of the 1.4 million PLHIV in Mozambique received continuous ART. ${ }^{2}$

Option $\mathrm{B}+$ is the most recent approach to PMTCT in low-resource settings. ${ }^{3-11}$ As an expansion of widespread treatment, all pregnant women diagnosed with HIV are initiated on a lifelong triple regimen of ART, regardless of CD4 count. ${ }^{10}$ In 2013, Mozambique initiated the rollout of Option B+. By 2015, Mozambique plans to provide Option $\mathrm{B}+$ in $50 \%$ of the country's 1,414 health centers. ${ }^{12}$ Given that the average fertility rate is 5.9 births per woman, with approximately 18 months between births, one motivation for the policy shift to Option B+ is to avoid repeated, short-term courses of ART during multiple pregnancies. ${ }^{12}$

Inherent to the design of Option $\mathrm{B}+$ is the recognition of WLHIV's fertility desires and continued intentions to become pregnant. Fertility desires of WLHIV are well documented in the literature. ${ }^{13-20}$ Fertility desires are positively associated with younger age, having fewer children, improved health status, and spouse and/or family support and/or pressure..$^{21,22}$ Evidence varies on whether or not the availability of ART increases the fertility desires of WLHIV. ${ }^{22-25}$ Factors associated with lessening fertility desires include fear of transmitting HIV to partners and children, negative effects of pregnancy on one's own health, and fear of negative reactions from health care workers. ${ }^{22}$

WLHIV often face stigma in clinical settings when they express their fertility desires and intentions to providers or become pregnant. ${ }^{14,19,26-30}$ In studies from Kenya, South Africa, and Uganda, participants did not discuss their fertility intentions with providers. ${ }^{13-15,30}$ The anticipation that they will experience stigma from providers also affects HIV-positive women's health care-seeking behaviors. In a study from rural Kenya, women had two times higher odds of planning to deliver at home if they feared maltreatment from clinic workers for being HIV-positive. ${ }^{31}$ In a different study from Kenya, women feared being mistreated by midwives if they used zidovudine during labor. ${ }^{32}$ A systematic review of the barriers and facilitators to PMTCT in sub-Saharan Africa found that staff/client interactions play a critical role and that negative staff attitudes are frequently cited as a barrier to women returning to health facilities. ${ }^{33}$

Stigma from health care workers against WLHIV's fertility intentions is also documented in studies with providers. ${ }^{34,35}$ In a study from Mozambique, providers adamantly discouraged their HIV-positive patients from becoming pregnant. ${ }^{34}$ In a study from Thailand, providers expressed the belief that PLHIV should not become pregnant due to concern for the welfare of the child if the parents should die. ${ }^{35}$
Contrastingly, a small minority of providers from the same studies acknowledged WLHIV's fertility desires and their options to have safe pregnancies. ${ }^{34,35}$ In a study from South Africa, providers even voiced concern that not acknowledging the fertility desires of WLHIV could cause women to disengage with treatment. ${ }^{36}$

In 2006, a US President's Emergency Plan For AIDS Relief (PEPFAR)-funded Positive Health, Dignity, and Prevention program targeting health care providers was introduced in Mozambique. This HIV prevention strategy highlights the needs of PLHIV and their role in the response to the global burden of HIV. In Mozambique, this strategy is called Prevençao Positiva (or Positive Prevention), which focuses prevention efforts on PLHIV, and training health care providers to build their own skills to assess behavioral and contextual risk among their patients and to provide a risk reduction-based prevention intervention focused on incremental change, tailored to the patient's transmission risk behavior and prevention needs. PMTCT and family planning are two key components of the Positive Prevention package in Mozambique. ${ }^{37,38}$

Similar to Positive Prevention, the success of Option $\mathrm{B}+$ requires effective provider participation. Provider stigma against WLHIV becoming pregnant could serve as a significant barrier to the success of Option B+. While provider views on the fertility desires and rights of WLHIV have been previously described in the literature, provider perspectives in the context of a Positive Prevention intervention have not yet been documented. This study was conducted as part of a larger qualitative evaluation to assess the feasibility and acceptability of Mozambique's Positive Prevention intervention. This paper adds to the literature by exploring provider descriptions of reproductive health messages as part of a Positive Prevention intervention in order to identity possible barriers and facilitators to successfully addressing family planning and pregnancy concerns of WLHIV.

\section{Materials and methods}

The components of the Mozambique Positive Prevention training and teaching methodologies have been described elsewhere, but briefly, the program consisted of a 3-day Positive Prevention training that was delivered over a 2-year period at several sites in three provinces (Maputo, Sofala, and Zambézia) where ART treatment was supported by PEPFAR. ${ }^{39}$ Key technical Positive Prevention components included an overview of the Positive Prevention model, risk reduction counseling, and prevention messages, discussing 
disclosure, family planning, PMTCT, and living positively, which is defined as taking action as it relates to one's health and well-being. The family planning component of the training focused on exploring the reasons why people have unintended pregnancies, the benefits of planning pregnancies, the connection between the risks for HIV and sexually transmitted infections and issues of family planning, long-term and short-term family planning methods, how health care providers can assist their HIV-positive patients to meet their family planning goals and practice discussing methods of family planning and negotiating sexual risk reduction or strategies to avoid an unintended pregnancy. The PMTCT section focused on strategies for WLHIV to reduce transmission of HIV from mother to child, challenges to effective PMTCT (including lack of access to medications, negative provider reactions to HIV-positive women becoming pregnant, social and cultural barriers, and the reluctance of health care providers to discuss pregnancy intentions in HIV care), values clarification/provider feelings around their patients being pregnant and how that can affect/harm the patient/provider relationship, the biomedical aspects of PMTCT, and identifying ways to equip health care workers with tools to support HIV-positive women who are pregnant or breastfeeding.

Individual in-depth interviews were conducted with 31 providers, all of whom had been trained in the Positive Prevention curriculum. Interviews were designed to provide a descriptive evaluation of the Positive Prevention training program and to ascertain how effective our training materials and approach were for providers, including which lessons providers learned and were able to implement in interactions with PLHIV, which Positive Prevention messages were difficult for them, and the applicability of training topics. While the main focus of the full study was assessing the feasibility and acceptability of Positive Prevention in Mozambique, the analysis for this paper focuses on a subset of the data related to provider comments on their feelings about and strategies to provide reproductive health care for PLHIV.

For this study, providers were defined as clinicians (including medical technicians and nurses), counselors (including counseling and testing staff, adherence support staff, support group leaders), and other site staff (such as pharmacists, laboratory technicians, and project management staff) who received the Positive Prevention training. Although their educational backgrounds and job functions differ, these various cadres were chosen to receive Positive Prevention training and to be evaluated because they represent the various types of health care workers that have contact with the patient population. To be eligible, providers had to be at least 18 years old, fluent in Portuguese, have participated in Positive Prevention training, and be regularly providing care to HIV-infected patients.

This sample consisted of five clinical care providers (two medical technicians and three nurses) and 26 non-clinical care providers (19 counselors or social workers, four peer educators, one project director, and two pharmacists or laboratory technicians). In Mozambique, there are only 3.3 doctors and 23.9 nurses per 100,000 people. ${ }^{40}$ Since there are so few clinical care providers in health centers, counselors and peers are a major part of the clinical staff. Counselors are the health educators and often act as the gatekeepers for information about family planning methods and how to have safer pregnancies. This sample size was chosen based on prior qualitative research activities conducted in Mozambique and the project budget. Although achieving saturation was not a criterion used to establish the sample size, saturation was observed during analysis through the consistency and redundancy of information during the interviews. ${ }^{41}$

Interviews were conducted at five rural Ministry of Health clinics within the three provinces where the Positive Prevention trainings took place. These sites included: the Namaacha Health Center and Esperança-Beluluane Counseling and Testing Center in Maputo Province, Mafambisse Health Center in Sofala Province, and the Namacurra Health Center and Inhassunge Hospital in Zambézia Province. Each site was chosen because it employed health care providers who had received the Positive Prevention training and was located in a province with both a high HIV prevalence and PEPFAR-funded treatment services. Providers were selected by the study staff using Positive Prevention training attendance lists. Providers who were interested in participating in the study gave their written informed consent prior to being interviewed.

Data collection took place from January through June 2010 and involved one round of interviews at each study site. Two sites were evaluated 2 years after receiving the Positive Prevention training (Namaacha Health Center and Esperanca-Beluluane Counseling and Testing Center in Maputo Province); one site was evaluated 6 months posttraining (Mafambisse Health Center in Sofala Province); and two sites (Namacurra Health Center and Inhassunge Hospital in Zambézia Province) were evaluated 2 months post-training. All interviews were conducted by trained interviewers in private rooms at the sites or in other private spaces on the 
site grounds. All interviews were conducted in Portuguese and were digitally recorded. All digital audio recordings of individual in-depth interviews were transcribed verbatim into Portuguese. A quality assurance protocol was built into the data management and analysis to monitor transcription accuracy. Transcripts were then translated into English. Back translation was used to verify the accuracy of the translation on a selected subsample of interviews. No names were disclosed during interviews. A participant code number was used to identify each audio tape and transcript, in place of names. ATLAS.ti qualitative analysis software (version 6.2, Scientific Software Development, Berlin, Germany, 2011) was used to manage the coding and analysis process.

Content analysis was utilized to interpret the data and allowed for answering the questions of this study. ${ }^{42}$ The qualitative analyst systematically worked through each transcript, identifying areas in which providers discussed the reproductive health or health care of WLHIV. Next, the analyst assessed the reproductive health data for pre-eminent themes and then determined codes. Codes included: WLHIV should not have children, condoms are the only valid form of family planning for WLHIV, pregnancy is proof that patients are not using condoms, if WLHIV already have children they should not have more, having a lot of children causes poor health outcomes for WLHIV, recognition of the importance of having children, WLHIV can safely have children, and protecting children as a prevention incentive. One researcher coded all the reproductive health data to ensure consistency.

The Committee on Human Research at University of California at San Francisco and the bioethics committee for the Mozambican Ministry of Health granted approval for this study.

\section{Results}

Thirty-one providers from five sites were interviewed. Fiftyfive percent of participants were female and $45 \%$ were male. The majority of participants were non-clinical care providers and $61 \%$ of all participants were counselors or social workers. Further provider demographic characteristics are shown in Table 1.

The providers' perspectives on family planning, pregnancy, and PMTCT messages in Positive Prevention were diverse and could be arranged along a continuum (Figure 1). At one end of the continuum is the message that WLHIV should not become pregnant. Towards the middle, providers are presenting reproductive health messages in more positive ways, such as recognition of the importance of having children. At the other end of the continuum is
Table I Provider demographics $(n=31)$

\begin{tabular}{ll}
\hline Characteristic & $\begin{array}{l}\text { Total number of providers } \\
\mathbf{n}=\mathbf{3} \text { I (\%) }\end{array}$ \\
\hline $\begin{array}{l}\text { Gender } \\
\text { Female }\end{array}$ & $17(55)$ \\
Male & $14(45)$ \\
Language & $31(100)$ \\
$\quad$ Portuguese & \\
Age & $8(26)$ \\
Under 30 & $16(52)$ \\
$30-39$ & $7(39)$ \\
$40+$ & $12(39)$ \\
Interview provinces & $10(32)$ \\
Zambézia & $9(29)$ \\
Sofala & \\
Maputo & \\
Occupation & $3(10)$ \\
Clinical care providers & $2(6)$ \\
Nurse & $19(61)$ \\
Medical technician & $4(13)$ \\
Non-clinical care providers & $2(6)$ \\
Counselor/social worker & $1(3)$ \\
Peer educator & \\
Pharmaceutical technician & \\
Project director of community & \\
education health group &
\end{tabular}

an example of one provider who described how she uses women's desires to protect their children as a clinical prevention incentive. Most messages from providers are on the negative end of the continuum and are framed as absolutes.

\section{WLHIV should not get pregnant - condom use is the only valid form of family planning}

Most providers articulated that they believed HIV-positive women should not have children and advised their HIVpositive patients not to become pregnant. They explained that they also believed condom use is the only valid form of family planning for women living with HIV and that other family planning methods do not have a place in HIV care. Their reasons for discouraging or withholding alternative forms of contraception from WLHIV ranged from the belief that women would forget to take birth control pills to the idea that condoms are the safest form of family planning.

"I have advised them ... it is better to have sex [with condoms] and to adopt a child, you have to always use condoms and each couple needs to be followed more." (Female coordinator, age 39 years, Sofala Province)

"I have seen women do family planning. When a woman is on ART, I usually tell them it's not good to do family planning, because one day they may be careless and get 


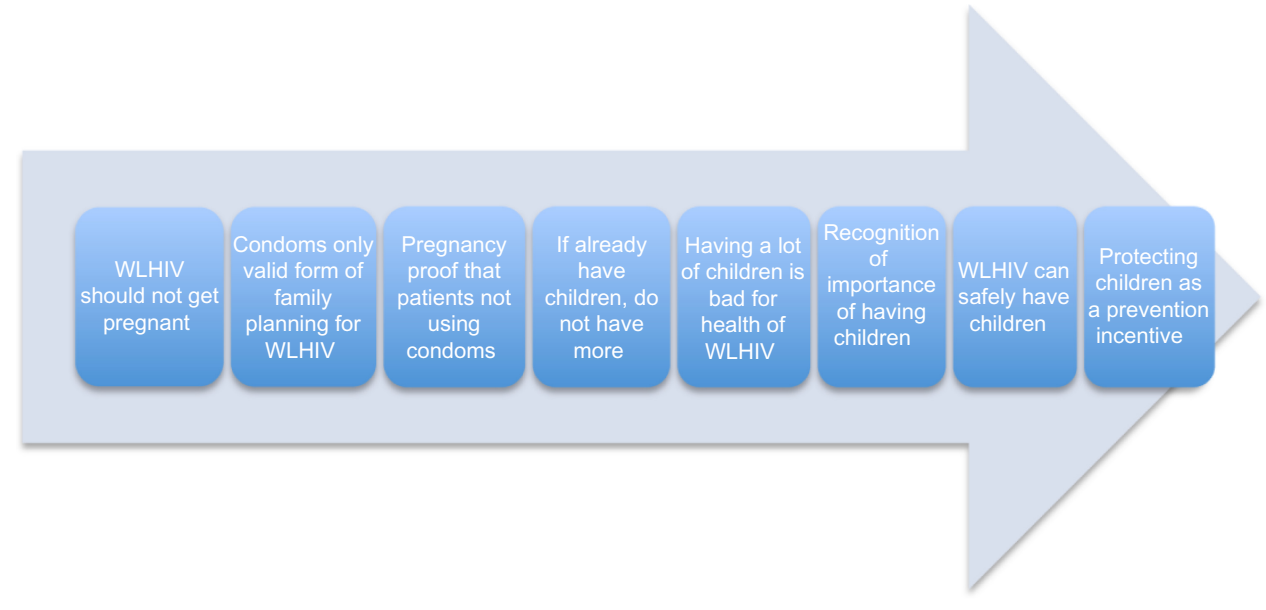

Figure I Continuum of reproductive health messages in provider narratives. Abbreviation: WLHIV, women living with HIV.

pregnant and that's not good. In this case, it is best to use a condom which is reassuring. Yes ... The important thing here in the clinic is to use condoms, here we advise people to use condoms. Because we did not focus much on family planning, because it can be neglected, yes ... The focus here is the use of condoms, yes." (Male counselor, age 39 years, Zambézia Province)

"This [counseling or talking with patients] is for prevention, family planning perhaps maybe we could also talk about condoms, which is a form of family planning, it is safer. The safest and most positive impact as well. Pills are more to prevent pregnancy, and not for HIV." (Male pharmacist, age 27 years, Maputo Province)

"Every woman that wants to do family planning, pill or injection, first we have them do an [HIV] test, they get tested and then after the test if the results come out negative then we give them the method to do." (Female counselor, age 35 years, Zambézia Province)

These quotes represent one end of the continuum and indicate that providers hold strong beliefs about the reproductive health capacities of WLHIV and that these providers translate those beliefs into Positive Prevention clinical messages. Providers expressed the opinion that WLHIV should not have children. Within these provider accounts, discussions of how to have safe pregnancies were completely absent. These expressions indicate that providers focus their family planning Positive Prevention messages on condom use because they prioritize sexual transmission to others over the reproductive health needs of their patients. Despite Positive Prevention training on the efficacy of treatment as prevention and the desire and right of WLHIV to have children, the majority of providers have not integrated this message into their clinical beliefs or practices in regard to vertical and sexual transmission.

\section{Pregnancy as proof that patients were not using condoms}

Providers discussed how they used pregnancy as an indicator of whether or not patients used condoms. If an HIV-positive woman arrived at the health center pregnant, providers said that her pregnancy proved she had not followed their instructions to always use condoms. Conversely, providers articulated that they interpreted a lack of pregnancy as proof that their patients had been consistently using condoms.

"We discover when, for example, a patient is on ... antiretroviral treatment, and one day they already have a belly, so we discover that this patient was not using condoms ... we discovered that they do not adopt a family planning method, if for example, they do not use condoms because they got pregnant, yes because if they are not pregnant at least you know, we can say yes, this one is using condoms." (Female counselor, age 35 years, Zambézia Province)

"We're working in a world with patients from a lower level, so they have that thing that family is their wealth and children, and they want to have children, they do not accept using condoms, you know that sort of thing ... It's difficult ... they do not change, they need to use prevention, condom use ... We can counsel a patient for two, three months, they show up pregnant. So that means that they do not use condoms. It's difficult, I think that we'll never be able to fight." (Female counselor, age 32 years, Zambézia Province)

"They always say they will use prevention, you know, they always say they will protect themselves, they always say oh we use condoms, and it is not always true, because many patients who we have had here, for the women, we talked about using condoms, using condoms with your partner, and the next month they show up pregnant ... I have seen here, in the day to day when we talk to the patient, I am 
going to give you an example of a woman, you know, that is in a program ... in the ARV department, we talked about methods of prevention, the risk that you run and if you get pregnant, the risk that the baby runs, but months later, as I was saying, they show up pregnant." (Female counselor, age 33 years, Zambézia Province)

These statements illustrate how providers saw a direct connection between condom use and pregnancy. Pregnancy, or lack thereof, indicated whether or not patients used condoms as they were instructed to do and demonstrated that providers were not prioritizing alternative methods for family planning.

\section{IfWLHIV already have children, they should not have more}

Providers described how they encouraged WLHIV who already had living children to use family planning methods in order to avoid further pregnancies. They discussed how they first inquired whether or not HIV-positive women had children and how the patient's response directed their clinical course of action.

"There are a lot of mothers who are now encouraged to do family planning. Some still have problems, because they still have the problem that her husband is the one who makes decisions and she does not decide. But most are doing family planning. We also counsel them, we ask how many children do you have, she answers, and we ask, you do not want to do planning. You have to do planning, because your condition is this. We counsel them ... In the first place, the type of planning that I recommend is to use condoms. Second, for a mother who has more than four children, I advise her to use the DEP [Depo-Provera]." (Female counselor, age 32 years, Zambézia Province)

"For those people who already have children we just tell them to use condoms, because it is very difficult with our community, we have had many problems, many people do not want to use condoms even though they are HIV positive, so if at least they do family planning to avoid pregnancy that would be good because having more children with this situation will not help them very much." (Female program manager, age 33 years, Sofala Province)

These quotes tell the story that clinicians focused specific family planning messages on women who have children, discouraging them from having more children. None of these accounts included discussions of WLHIV's continued fertility desires and/or the possible childbearing expectations of women, their partners, families, and/or communities. Moving along the continuum, although these providers stated that they primarily promoted condom use, they at least offered their patients other methods of family planning. While these statements indicate that providers did offer patients family planning methods other than condoms, there is no discussion about offering women a range of methods or letting a woman choose which method is right for her.

Other providers spoke about encouraging WLHIV who have living children to delay and purposefully space future pregnancies as a means of protecting the health of both mother and children.

"Family planning is very good for a patient. Because, for example, eh ... Supposing that we have HIV-positive mothers, that mother needs to do planning because their ... CD4 count is very low, this can create a risk during childbirth. These are risks that we say, we teach our patients ... For example we pick up those ladies [who] have one child born in one year and another in another year, you know, for example, she conceived now, will conceive again, I explain to her, but if you do planning you know that João is already five, I'm going to have another baby, João doesn't have many problems, and no longer has many risks, because he can already ask for water, he can already do something, you can already take care of his health." (Female coordinator, age 39 years, Sofala Province)

"When they realize they are HIV positive and we communicate that family planning is the best option and prolongs life. Because when a person does not do family planning, for example if a woman does not do family planning and she produces many children she runs the risk of worsening their condition and being more frail. Then by explaining it and her realizing it, I think this is one of the things that facilitates this activity [adopting family planning methods]." (Female medical technician and director of Namaacha Health Center, age 44 years, Maputo Province)

These expressions differ from those above where providers described discouraging WLHIV who already had children from becoming pregnant. In contrast, these providers explained that they were discouraging patients from becoming pregnant as a method of birth spacing to promote maternal and child health. These messages are framed in a more positive manner and move along the continuum.

\section{Recognition of the importance of having children}

Some providers explained that they understood the personal and cultural importance of having children. They expressed a 
recognition of the challenges faced by WLHIV in reconciling their fertility desires with their HIV status. Providers also noted that WLHIV chose not to use contraceptives in order to become pregnant.

"It depends on the age whether they do family planning or not. They say they are still young. So when they are young they cannot die without children.” (Male medical technician, age 47 years, Maputo Province)

"In the case of young people we inform them of condom use and they avoid it to get pregnant, many times people do not comply ... We are in Africa and here people marry to have children, so people know that by using condoms they will not get pregnant and they will not have kids." (Male counselor, age 26 years, Zambézia Province)

"It is very difficult, there are people who are positive but they want to have a child. How can we have a couple, they need to have a child but with HIV/AIDS they stop. It's enough that they want to die, because they want to have a child." (Female voluntary counseling and testing counselor, age 60 years, Sofala Province)

Dissimilar to the quotes in the previous theme, these expressions indicate that some providers acknowledged both the fertility desires of WLHIV and the cultural expectations of childbearing. In this theme, providers acknowledged the role of childbearing in the cultural context of WLHIV's lives. This recognition, and the absence of stigma-based opinions within these statements, represents a considerable shift along the continuum of reproductive health messages.

\section{PLHIV can safely have children - using fertility desires as a prevention incentive}

A few providers told the story of working with WLHIV to have safe pregnancies. They explained that they told their HIV-positive patients that WLHIV could safely have children, but that they must first improve their own health and then work with doctors in order to minimize vertical transmission risks. A small number of providers even spoke about using WLHIV's fertility desires as a clinical prevention tool to encourage patients to adhere to ART.

"What is difficult to talk about is condom use for a couple infected with HIV who wants to have children. But we tell them that they should not go forward with having children while in a fragile state, it would be better after improving their physical condition and recovering, we have to regain health and then go back to how to... They say, but we will not have children for the rest of our lives? You can have children but there are clinical criteria. And we inform them no, when they are physically recovered, the doctors will see how to follow the right steps. First we have to protect the disease, and once the disease has recovered then you can have children." (Male counselor, age 39 years, Zambézia Province)

"Others have demonstrated a difficulty of 'if I use condoms then I can't have kids?' You have to help them and inform them, the impact, having a child is not a good idea while you are in a chaotic state. First, worry about maintaining your good health. Some have the future concern of children but the doctors can get involved ... find out with them how you can have a child while living positively.” (Male counselor, age 35 years, Zambézia Province)

"We also know that everybody wants to have children, many young people who were unable to have children became infected before having children, then the advice that we give to the person is to look for a means, that is, know their condition in relation to their $\mathrm{CD} 4$, find someone who will help you know if you can get pregnant or not." (Female program manager, age 33 years, Sofala Province)

"When I'm dealing with, some young people who still do not have children, let's suppose, then sometimes they ask me questions, 'even when we have children, are we going to die this way?' and I encourage ... 'Life is not going to end this way.'... if you can improve the health status in this case, if the viral load began to decrease, then the doctor is going to assess when the people come, for example, the doctor orders the tests to be done, then from there, you consult your doctor if you can have children or not, so it is a trick that I think encourages them." (Female maternal and child health nurse, age 43 years, Maputo Province)

Moving along the continuum, these quotes illustrate that some providers not only recognized WLHIV's fertility desires, but that their clinical messages included the ability of WLHIV to have children and strategies of how to have a safe pregnancy. These strategies included improving one's own health before becoming pregnant and working with providers to determine the safest time to become pregnant. A few providers told the story of using WLHIV's fertility desires as an incentive to engage in care and adhere to treatment. These quotes are a unique example of providers using the importance of having children as a tool to encourage Positive Prevention. Looking along the continuum, the number of quotes that positively frame WLHIV's fertility desires and capacities is small. Negative reproductive health messages are more dominant. These quotes offer an opportunity to rephrase reproductive health messages in the context of HIV clinical care. 


\section{Discussion}

These data portray multiple perspectives that providers report using in Mozambique to address pregnancy desires and family planning as one component of the Positive Prevention program, and that these perspectives flow along a continuum. Many reproductive health messages described by providers demonstrate the narrow view that they take in addressing family planning with WLHIV. Providers expressed that WLHIV should not become pregnant, condoms are the only valid form of family planning for WLHIV, pregnancy is proof that WLHIV do not use condoms, and if WLHIV already have children then they should not have more. A smaller number of messages demonstrated an understanding and recognition of the importance of having children and a wider range of strategies for WLHIV to have safe pregnancies, and thus suggested different ways in which the desire for children could be supported.

Previous studies have also shown that providers believe WLHIV should not become pregnant. ${ }^{34,35}$ In a survey from Gaza Province, Mozambique, clinic staff were insistent in their recommendations to HIV-positive women that they cease childbearing indefinitely. ${ }^{34}$ Our findings are also consistent with a study from Kenya, conducted from the patient perspective, which found that HIV-positive men and women reported that their providers had strongly encouraged them to stop childbearing and had offered condoms as the only option for family planning. ${ }^{30}$ Despite what was taught in the Positive Prevention training, many providers had preconceived ideas about WLHIV becoming pregnant and what they should and should not do. ${ }^{43}$

Similarly, although the definition of family planning, as taught in the Positive Prevention curriculum, was broad and included both long-term and short-term methods, most providers also communicated that condoms are the only valid form of family planning for WLHIV. ${ }^{43}$ Only offering condoms to WLHIV severely limits women's abilities to protect themselves from unintended pregnancies. Providers who view condoms as the safest form of contraception overlook the risks some women face in negotiating condom use with their partners. Barriers to WLHIV successfully negotiating condom use with their partners include unequal gender relationships, men's outright refusal, lack of condom use between primary partners, and fear of violence if condom use indicates disclosure of HIV status. ${ }^{44} 46$

A provider assuming that pregnancy is proof that patients were not using condoms is highly problematic for a variety of reasons. First, absence of pregnancy is not necessarily an accurate indicator of consistent condom use. In a study from Uganda, WLHIV stated that they often exaggerated their condom use when talking to providers. ${ }^{13}$ Second, absent from these expressions is any acknowledgment that patients may have actively chosen to forego condom use in order to become pregnant. In a study from the USA, HIVpositive women said they actively chose to not use condoms in attempts to conceive. ${ }^{47}$ Third, these provider reactions to pregnancy could serve as a barrier to both men and women who want to access PMTCT services and engage in care. In a study from Malawi, an antenatal attendee explained that she feared returning to the health facility for nevirapine syrup after giving birth because she anticipated that the staff would criticize her for giving birth at home instead of in the health facility. ${ }^{48}$ If WLHIV expect to be shamed or are made to feel bad by providers when they present pregnant at health facilities, they may not return to health facilities for antenatal or post partum care services. This therefore puts the woman's health, and the health of her children, at risk.

Moving along the continuum, some providers framed their messages on pregnancy and family planning from a more positive, patient-centered, reproductive rights perspective. Advising patients to space pregnancies is an evidence-based clinical message. The World Health Organization recommends a 2-year interval between a live birth and the next pregnancy attempt in order to reduce the risk of poor maternal, perinatal, and infant health outcomes. ${ }^{49}$ Advocating birth spacing to patients was also taught in the Positive Prevention curriculum. ${ }^{43}$

Some providers articulated their understanding that having children is a tenet of Mozambican society. In previous studies from Kenya, South Africa, and Uganda, WLHIV described feeling pressure to continue childbearing from their partners, families, and communities..$^{13-15,30}$ Recognizing the cultural expectation of childbearing is important for providers to be able to appreciate the pressure that their patients might be under to continue childbearing despite clinical recommendations.

A few providers offered patients two main strategies for WLHIV to have safe pregnancies, ie, improving one's own health before becoming pregnant and working with doctors to determine the safest time to become pregnant. Advising patients to improve their own health prior to conception by adhering to ART and attempting to achieve an undetectable viral load is the foundation of preconception care for WLHIV. $^{50,51}$ Vertical transmission rates are significantly lower for women who become pregnant while on ART compared with women who initiate ART during pregnancy. ${ }^{52}$

While the first recommendation of improving the mother's health prior to conception is evidence-based, the second 
recommendation of consulting with a doctor is problematic given the lack of physicians in the clinics in which these providers work, as also previously noted in a similar study with providers from Mozambique. ${ }^{34}$ In the clinics in which this study was carried out, there is typically only one medical doctor on site. This lone physician is often the medical director of the facility, and works in an administrative and not a clinical capacity. Shifting the responsibility for discussions about safe pregnancies onto providers with higher authority denies WLHIV access to information as such providers are often absent.

While most of the statements from providers disregarded WLHIV's fertility desires, fertility intentions, and reproductive rights, a few providers discussed using their patients' desires to protect their children as a clinical prevention incentive. To our knowledge, this strategy is not well documented in the literature. Building on using WLHIV's fertility desires and intentions as clinical prevention incentives in countries where fertility rates are high could improve adherence to ART and uptake of family planning.

This study had several limitations. The data for this analysis may have been affected by social desirability bias because the data came from provider interviews. To minimize this potential bias, interviewers were hired study staff and were not affiliated with the Positive Prevention training program or the Ministry of Health. Also, these data were self-reported and we were not able to validate provider reports (for example, by triangulating with data from their actual clients). Although the length of time between when providers received the Positive Prevention training and when the interviews took place varied by province, responses from providers did not vary significantly across sites. Another limitation of this study is that the interview guide was not designed with this research question in mind. Therefore, it is possible that we are missing certain nuances about this topic that providers would have discussed further had the interview guide probed further. Further, only one researcher conducted the data coding. Although using only one coder creates consistency, it also brings subjectivity, which could be a limitation of our data.

The majority of reproductive health messages described by providers represent possible barriers to the rollout of Option $\mathrm{B}+$ and could serve as a significant obstacle to the success of this strategy in Mozambique. Providers' preconceived ideas toward WLHIV's reproductive intentions and family planning options might be a result of stigma. Previous studies have defined HIV-related stigma as a barrier to women accessing maternity care and clinic workers providing care. ${ }^{27,28,31,53-56}$ Turan et al found that stigma negatively affects the ways in which clinic workers provide labor and delivery services for HIV-positive women or women they suspect of being HIV-positive. ${ }^{56}$ In a review of the literature on the effects of HIV-related stigma on PMTCT, Turan and Nyblade concluded that stigma negatively impacts all phases of PMTCT, including attendance at antenatal services, adherence to ART during pregnancy and postpartum, and breastfeeding practices. ${ }^{28}$ Our findings indicate that providers did not support WLHIV to exercise their full reproductive rights, possibly due to stigma and discomfort with advising WLHIV about getting pregnant and the risks of vertical transmission. A woman, not her clinician, should determine the number of children that she wants to have and the method of family planning that she wants to use.

Addressing provider stigma and biases about HIV-positive women becoming pregnant and about access to a range of safe and effective family planning methods is essential. Despite the attention to family planning in the Positive Prevention curriculum, there is no shift between our findings on providers' views and those of Hayford and Agadjanian in 2010. ${ }^{34}$ The family planning and PMTCT training that the providers received, as part of a 3-day training, was clearly insufficient to truly change the feelings of providers about pregnancy and family planning for WLHIV. Beliefs about pregnancy and chronic disease may be very ingrained. Although treatment is available and WLHIV can have safe pregnancies, many providers might still be focused on a time when HIV was a death sentence and have not yet made a shift in their mindset. This indicates the need for an intensive approach, ie, interventions specifically focused on family planning in HIV clinic settings.

The Positive Prevention curriculum can be improved by emphasizing reproductive health services that are free from judgment, where women are supported to have safe and healthy pregnancies should they so choose and have access to a full range of family planning options that meet their needs (and not the needs of their care providers). To move providers along the continuum, we suggest conducting more training with providers that focuses on values clarification. The rollout of Option B+ should include training for providers on how to communicate with WLHIV on reproductive issues and childbearing intentions and how to recognize their own biases. Trainings could also focus on advising providers to let patients make their own reproductive decisions or trying to discuss reproductive intentions with patients sooner. Providers also need more training in family planning methods that are appropriate for WLHIV.

In addition to addressing provider prejudice, change must be effected at the institutional level. Further efforts 
need to be made to integrate family planning into the HIV clinical consultation work. In order to succeed, providers need structural reinforcements to improve family planning for WLHIV. While clinical protocols for integrating Positive Prevention into health facilities in Mozambique have been approved by the Ministry of Health and are in place, little attention has been focused on supporting clinics to integrate family planning into HIV care. Appointment prompts and reminders could prioritize family planning needs and fertility intentions and potentially result in an increase of providers addressing these needs for WLHIV. In addition, health facilities in Mozambique often experience stock-outs of popular family planning methods. ${ }^{57}$ Health facilities need to ensure that there is a steady and varied supply of family planning methods for women to choose from, and this issue needs to be addressed not only at the health facility but also at the country supply-chain level. The reproductive health and rights of WLHIV also need to be prioritized at the policy level. Developing national guidelines on how to integrate family planning and reproductive health services into HIV care could help draw national attention to the issue and improve reproductive services for people living with HIV throughout Mozambique.

Our findings indicate an uphill battle for the success of Option $\mathrm{B}+$ in Mozambique. Inherent to the design of Option $\mathrm{B}+$ is the acknowledgment that a pregnant, HIVpositive woman presenting at antenatal care is likely to become pregnant again. Moving providers along the continuum, to be more positive and accepting about the reproductive desires of WLHIV, would help support Option B+ in Mozambique. Providers could utilize the importance of having children as a point of access to address both Positive Prevention and the pregnancy desires of individual patients in this high HIV prevalence setting. Reframing reproductive health messages in Positive Prevention is an opportunity to address the needs of individuals, their partners, their children, and the larger community epidemic.

\section{Disclosure}

The authors report no conflicts of interest in this work.

\section{References}

1. National Institute of Health, National Institute of Statistics, ICF Marco. National Survey on Prevalence, Behavioral Risks and Information about HIV and AIDS in Mozambique 2009. Calverton, MD:2010.

2. World Health Organization, Joint United Nations Programme on HIV/AIDS, United Nations Children's Fund. Scaling up priority HIV/AIDS interventions in the health sector. 2010. Available from: http://www.who.int/hiv/pub/2010progressreport/report/en/. Accessed August 17, 2014.
3. Schouten EJ, Jahn A, Midiani D, et al. Prevention of mother-to-child transmission of HIV and the health-related Millennium Development Goals: time for a public health approach. Lancet. 2011;378(9787):282-284.

4. Centers for Disease Control and Prevention. Impact of an innovative approach to prevent mother-to-child transmission of HIV - Malawi, July 2011-September 2012. MMWR Morb Mortal Wkly Rep. 2013;62(8): $148-151$.

5. Coutsoudis A, Goga A, Desmond C, Barron P, Black V, Coovadia H. Is Option B+ the best choice? Authors' reply. Lancet. 2013;381(9874): 1273-1274.

6. Coutsoudis A, Goga A, Desmond C, Barron P, Black V, Coovadia H. Is Option B+ the best choice? Lancet. 2013;381(9863):269-271.

7. Goosby EP. Is Option B+ the best choice? Lancet. 2013;381(9874):1272.

8. Hirnschall G, Doherty M, Shaffer N. Is Option B+ the best choice? Lancet. 2013;381(9874):1271-1272.

9. Kellerman S, Jay JS, Quick JD. Is Option B+ the best choice? Lancet. 2013;381(9874):1273.

10. World Health Organization. Use of antiretroviral drugs for treating pregnant women and preventing HIV infection in infants - Programmatic update. 2012. Available from: http://www.who.int/hiv/pub/mtct/ programmatic_update2012/en/. Accessed August 17, 2014.

11. Schouten EJ, Jahn A, Chimbwandira F, Harries AD, Van Damme W. Is Option B+ the best choice? Lancet. 2013;381(9874):1272-1273.

12. Ministry of Health. HIV/AIDS acceleration plan 2013-2015. Maputo, Mozambique: Ministry of Health; 2014.

13. Beyeza-Kashesya J, Kaharuza F, Mirembe F, Neema S, Ekstrom AM, Kulane A. The dilemma of safe sex and having children: challenges facing HIV sero-discordant couples in Uganda. Afr Health Sci. 2009;9(1):2-12.

14. Cooper D, Harries J, Myer L, Orner P, Bracken H. "Life is still going on": reproductive intentions among HIV-positive women and men in South Africa. Soc Sci Med. 2007;65(2):274-283.

15. Cooper D, Moodley J, Zweigenthal V, Bekker LG, Shah I, Myer L. Fertility intentions and reproductive health care needs of people living with HIV in Cape Town, South Africa: implications for integrating reproductive health and HIV care services. AIDS Behav. 2009;13 Suppl 1:38-46.

16. Kakaire O, Osinde MO, Kaye DK. Factors that predict fertility desires for people living with HIV infection at a support and treatment centre in Kabale, Uganda. Reprod Health. 2010;7:27.

17. Kanniappan S, Jeyapaul MJ, Kalyanwala S. Desire for motherhood: exploring HIV-positive women's desires, intentions and decisionmaking in attaining motherhood. AIDS Care. 2008;20(6):625-630.

18. Mmbaga EJ, Leyna GH, Ezekiel MJ, Kakoko DC. Fertility desire and intention of people living with HIV/AIDS in Tanzania: a call for restructuring care and treatment services. BMC Public Health. 2013;13:86.

19. Nattabi B, Li J, Thompson SC, Orach CG, Earnest J. Between a rock and a hard place: stigma and the desire to have children among people living with HIV in northern Uganda. J Int AIDS Soc. 2012;15(2):17421.

20. Wagner G, Linnemayr S, Kityo C, Mugyenyi P. Factors associated with intention to conceive and its communication to providers among HIV clients in Uganda. Matern Child Health J. 2012;16(2):510-518.

21. McClellan MK, Patel R, Kadzirange G, Chipatod T, Katzenstein D. Fertility desires and condom use among HIV-positive women at an antiretroviral roll-out program in Zimbabwe. Afr J Reprod Health. 2010;14(2):27-35.

22. Nattabi B, Li J, Thompson SC, Orach CG, Earnest J. A systematic review of factors influencing fertility desires and intentions among people living with HIV/AIDS: implications for policy and service delivery. AIDS Behav. 2009;13(5):949-968.

23. Berhan Y, Berhan A. Meta-analyses of fertility desires of people living with HIV. BMC Public Health. 2013;13(1):409.

24. Kipp W, Heys J, Jhangri GS, Alibhai A, Rubaale T. Impact of antiretroviral therapy on fertility desires among HIV-infected persons in rural Uganda. Reprod Health. 2011;8:27.

25. Maier M, Andia I, Emenyonu N, et al. Antiretroviral therapy is associated with increased fertility desire, but not pregnancy or live birth, among $\mathrm{HIV}+$ women in an early HIV treatment program in rural Uganda. AIDS Behav. 2009;13 Suppl 1:28-37. 
26. Orner P, Cooper D, Myer L, Zweigenthal V, Bekker LG, Moodley J. Clients' perspectives on HIV/AIDS care and treatment and reproductive health services in South Africa. AIDS Care. 2008;20(10):1217-1223.

27. Rahangdale L, Banandur P, Sreenivas A, Turan JM, Washington R, Cohen CR. Stigma as experienced by women accessing prevention of parent-to-child transmission of HIV services in Karnataka, India. AIDS Care. 2010;22(7):836-842.

28. Turan JM, Nyblade L. HIV-related stigma as a barrier to achievement of global PMTCT and maternal health goals: a review of the evidence. AIDS Behav. 2013;17(7):2528-2539.

29. Kendall $\mathrm{T}$. Reproductive rights violations reported by Mexican women with HIV. Health Hum Rights. 2009;11(2):77-87.

30. Withers M, Dworkin S, Harrington E, et al. Fertility intentions among HIV-infected, sero-concordant couples in Nyanza province, Kenya. Cult Health Sex. 2013;15(10):1175-1190.

31. Medema-Wijnveen JS, Onono M, Bukusi EA, Miller S, Cohen CR, Turan JM. How perceptions of HIV-related stigma affect decision-making regarding childbirth in rural Kenya. PLoS One. 2012;7(12):e51492.

32. Kiarie JN, Kreiss JK, Richardson BA, John-Stewart GC. Compliance with antiretroviral regimens to prevent perinatal HIV-1 transmission in Kenya. AIDS. 2003;17(1):65-71.

33. Gourlay A, Birdthistle I, Mburu G, Iorpenda K, Wringe A. Barriers and facilitating factors to the uptake of antiretroviral drugs for prevention of mother-to-child transmission of HIV in sub-Saharan Africa: a systematic review. J Int AIDS Soc. 2013;16(1):18588.

34. Hayford SR, Agadjanian V. Providers' views concerning family planning service delivery to HIV-positive women in Mozambique. Stud Fam Plann. 2010;41(4):291-300.

35. Ko NY, Muecke MA. Prevailing discourses among AIDS care professionals about childbearing by couples with HIV in Taiwan. AIDS Care. 2006;18(1):82-86.

36. Harries J, Cooper D, Myer L, Bracken H, Zweigenthal V, Orner P. Policy maker and health care provider perspectives on reproductive decision-making amongst HIV-infected individuals in South Africa BMC Public Health. 2007;7:282.

37. Dawson Rose C, Gutin SA, Reyes M. Adapting positive prevention interventions for international settings: applying US evidence to epidemics in developing countries. J Assoc Nurses AIDS Care. 2011;22(1):38-52.

38. University of California San Francisco. The acceptability and feasability of positive prevention in Mozambique. 2011. Available from: http://positiveprevention.ucsf.edu/CDROM/HTML/PDF/Policy_Brief_ En.PDF. Accessed August 17, 2014.

39. Gutin SA, Cummings B, Jaiantilal P, Johnson K, Mbofana F, Rose CD Qualitative evaluation of a Positive Prevention training for health care providers in Mozambique. Eval Program Plann. 2014;43C:38-47.

40. Republic of Mozambique Council of Ministers. National Stategic HIV and AIDS Response Plan2010-2014. 2009. Available from: https://www.google. co.nz/webhp? sourceid=chrome-instant\&ion $=1 \&$ espv $=2 \&$ ie $=U T F-$ $8 \# q=$ Republic $\% 20$ of $\% 20$ Mozambique $\% 20$ Council $\% 20$ of $\% 20$ Ministers.\%20National\%20Stategic\%20HIV\%20and\%20AIDS\%20 Response\%20Plan. Accessed August 17, 2014.

41. Pope C, Ziebland S, Mays N. Qualitative research in health care Analysing qualitative data. BMJ. 2000;320(7227):114-116.

42. Charmaz K. Premises, principles, and practices in qualitative research: revisiting the foundations. Qual Health Res. 2004;14(7):976-993.
43. University of California San Francisco. Mozambique Positive Prevention Training: Facilitator Guide and Participant Manual (Version 1.4). San Francisco, CA: University of California, San Francisco School of Nursing; 2009. Available from: PositivePrevention.ucsf.edu. Accessed September 24, 2014

44. Allen C, Mbonye M, Seeley J, et al. ABC for people with HIV: responses to sexual behaviour recommendations among people receiving antiretroviral therapy in Jinja, Uganda. Cult Health Sex. 2011;13(5): 529-543.

45. Dworkin SL, Ehrhardt AA. Going beyond "ABC" to include "GEM": critical reflections on progress in the HIV/AIDS epidemic. Am J Public Health. 2007;97(1):13-18.

46. Pettifor A, MacPhail C, Corneli A, et al. Continued high risk sexual behavior following diagnosis with acute HIV infection in South Africa and Malawi: implications for prevention. AIDS Behav. 2011;15(6): 1243-1250.

47. Finocchario-Kessler S, Mabachi N, Dariotis JK, Anderson J, Goggin K, Sweat M. "We weren't using condoms because we were trying to conceive": the need for reproductive counseling for HIV-positive women in clinical care. AIDS Patient Care. 2012;26(11):700-707.

48. O'Gorman DA, Nyirenda LJ, Theobald SJ. Prevention of motherto-child transmission of HIV infection: views and perceptions about swallowing nevirapine in rural Lilongwe, Malawi. BMC Public Health. 2010;10:354.

49. World Health Organization. Report of a WHO Technical Consultation on Birth Spacing: Geneva, Switzerland 13-15 June 2005. 2006. Available from: http://www.who.int/maternal_child_adolescent/ documents/birth_spacing.pdf. Accessed August 17, 2014.

50. Aaron E, Criniti S. Preconception health care for HIV-infected women Top HIV Med. 2006;15(4):137-141.

51. Bekker L, Black V, Myer L, et al. Guideline on safer conception in fertile HIV-infected individuals and couples. South Afr J HIV Med. 2011;12(2):31-44

52. Hoffman RM, Black V, Technau K, et al. Effects of highly active antiretroviral therapy duration and regimen on risk for mother-to-child transmission of HIV in Johannesburg, South Africa. J Acquir Immune Defic Syndr. 2010;54(1):35-41.

53. Cuca YP, Onono M, Bukusi E, Turan JM. Factors associated with pregnant women's anticipations and experiences of HIV-related stigma in rural Kenya. AIDS Care. 2012;24(9):1173-1180.

54. Turan JM, Bukusi EA, Onono M, Holzemer WL, Miller S, Cohen CR. HIV/AIDS stigma and refusal of HIV testing among pregnant women in rural Kenya: results from the MAMAS Study. AIDS Behav. 2011;15(6): $1111-1120$

55. Turan JM, Hatcher AH, Medema-Wijnveen J, et al. The role of HIV-related stigma in utilization of skilled childbirth services in rural Kenya: a prospective mixed-methods study. PLoS Med. 2012; 9(8): 1001295.

56. Turan JM, Miller S, Bukusi EA, Sande J, Cohen CR. HIV/AIDS and maternity care in Kenya: how fears of stigma and discrimination affect uptake and provision of labor and delivery services. AIDS Care 2008;20(8):938-945.

57. Chilundo B, Ortiz E, Jacinto A, Nhacale A. Supply- and demand-side barriers to use of contraception in three provinces of Mozambique. Presented at the International Conference on Family Planning, November 12-15, 2013, Addis Ababa, Ethiopia.
International Journal of Women's Health

\section{Publish your work in this journal}

The International Journal of Women's Health is an international, peerreviewed open-access journal publishing original research, reports, editorials, reviews and commentaries on all aspects of women's healthcare including gynecology, obstetrics, and breast cancer. The manuscript management system is completely online and includes

\section{Dovepress}

a very quick and fair peer-review system, which is all easy to use. Visit http://www.dovepress.com/testimonials.php to read real quotes from published authors. 